\title{
A New Diffractometer for High Energy Synchrotron Radiation at the Elliptical Multipole Wiggler at the APS
}

\author{
U. Ruett ${ }^{1.2}$, J. R. Schneider ${ }^{3}$, M. A. Beno', G. S. Knapp' and P. A. Montano ${ }^{1,4}$ \\ 'Materials Science Division, Argonne National Laboratory, Argonne, IL 60439 \\ ${ }^{2}$ Northern Illinois University, DeKalb, IL 60115 \\ ${ }^{3}$ Hamburger Synchrotron Strahiungslabor (HASYLAB), Hamburg, Germany \\ 'University of Chicago, Chicago, IL \\ Materials Science Division \\ Argonne National Laboratory
}

Argonne, IL 60439

\section{RECEIVED \\ SEP 211999 \\ OSTI}

The submitied manuscript has been created by
the University of Chicago as Operator of
Argonne National Laboratory (Algonne") Under
Contract No. W-31-109-ENG-38 with the U.S.
Department of Energy. The U.S. Govemment
retains for itself, and others acting on its
behalf, a paid-up, non exclusive, Irrevocable
wortdwide license in said article to reproduce,
prepare derivative works, distribute copies to
the public, and pertorm publicly and display
publicly, by or on behatf of the Govemment.

July 1998

jjc

Distribution:

1-2. PRS

3. B. D. Dunlap

4. P. A. Montano

5. F. Y. Fradin

6. R. Gottschall

7. Editorial Office

8. Authors

Submitted as a paper to be published in the proceedings of the SPIE 42nd Annual Meeting to be held July 19-24, 1998 in San Diego, CA.

This work is supported by the Division of Materials Sciences, Office of Basic Energy Sciences of DOE, under contract No. W-31-109-ENG-38. 


\section{DISCLAIMER}

This report was prepared as an account of work sponsored by an agency of the United States Government. Neither the United States Government nor any agency thereof, nor any of their employees, make any warranty, express or implied, or assumes any legal liability or responsibility for the accuracy, completeness, or usefulness of any information, apparatus, product, or process disclosed, or represents that its use would not infringe privately owned rights. Reference herein to any specific commercial product, process, or service by trade name, trademark, manufacturer, or otherwise does not necessarily constitute or imply its endorsement, recommendation, or favoring by the United States Government or any agency thereof. The views and opinions of authors expressed herein do not necessarily state or reflect those of the United States Government or any agency thereof. 


\section{DISCLAIMER}

Portions of this document may be illegible in electronic image products. Images are produced from the best available original document. 


\title{
A New Diffractometer for High Energy Synchrotron Radiation at the Elliptical Multipole Wiggler at the APS
}

\author{
U. Rütt 1,2, J. R. Schneider ${ }^{3}$, M. A. Beno', G. S. Knapp' and P.A. Montano ${ }^{1,4}$ \\ I Materials Science Division, Argonne National Laboratory, Argonne, IL 60439 \\ 2 Northern Illinois University, DeKalb, Illinois \\ ${ }^{3}$ Hamburger Synchrotron Strahlungslabor (HASYLAB) at DESY, Hamburg, Germany \\ ${ }^{4}$ University of Chicago, Chicago, Illinois
}

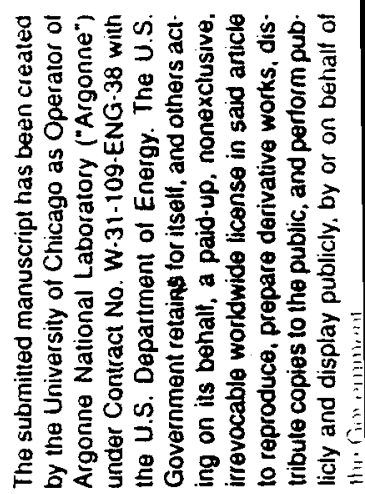

\begin{abstract}
The use of high energy synchrotron radiation (above $80 \mathrm{keV}$ ) for diffraction experiments offers many advantages resulting from the high penetration depth of the high energy photons and the small Bragg angles. The main features are: the possibility for the study of large sample crystals in transmission geometry, simple sample environments, high instrumental resolution in reciprocal space, the ability to utilize high momentum transfers and small correction factors for scattered intensities. The experiments performed at this kind of diffractometer are mainly flux experiments, in which the only requirement is a relatively small angular divergence for the incident beam in the scattering plane. The new triple crystal diffractometer introduced here will be installed at the elliptical multipole wiggler beamline at the Advanced Photon Source (APS). Because of the high critical energy of this device, $32 \mathrm{keV}$, the wiggler will produce high intensities at very high photon energies. To collect up to $1 \mathrm{mrad}$ of the horizontal divergence of the beam, a bent annealed silicon monochromator will scatter and focus in the horizontal scattering plane. The diffractometer will be operated in the vertical scattering plane taking advantage of the small vertical beam divergence.
\end{abstract}

Keywords: Synchrotron Radiation, Triple Crystal Diffractometer, Annealed Silicon Crystal, Bent Monochromator

\section{Introduction}

For the past decade high energy synchrotron radiation ( $E \geq 80 \mathrm{keV}$ ) has been used for diffraction experiments in a triple crystal set up. The first test diffractometer was operated on CHESS at Cornell University in $1989^{1}$. The first sophisticated diffractometer was built and has been used at HASYLAB since $1991^{2}$. At present three sophisticated diffractometers exist. Two instruments at HASYLAB utilize a high field wiggler beam line at the storage ring DORIS III ${ }^{2}$ and the undulator beam line at PETRA $\mathrm{II}^{3}$. The third instrument operates at a wiggler beamline on the ESRF ${ }^{4}$. The advantages of high photon energies result from weak interaction with matter and small wavelength. For example, the free mean beam path for silicon at $100 \mathrm{keV}$ is $23 \mathrm{~mm}$, for iron $3.5 \mathrm{~mm}$. Therefore, even large sample crystals, as used in material science studies, can be penetrated to study real bulk properties. Also sample environments, such as furnaces or cryostats, can easily be used with simple windows or in some cases without any windows. For most elements the electron binding energies are much smaller than $80 \mathrm{keV}$, so that generally no resonant scattering appears. Also the other correction factors such as extinction and polarization are small. The extinction length is proportional to the photon energy. In imperfect crystals the extinction effect is negligible or very small. Since for small momentum transfers the Bragg angles are only on the order of $2^{\circ}$, the polarization factor in this case is close to unity. At high energies the Bragg angle no longer limits the study of high momentum transfers. High energy photons offer the possibility for combining the high penetration power of neutron diffraction with the extremely high resolution reached with $\mathrm{X}$-ray scattering and the additional advantage of having only small correction factors for the scattered intensities. This technique has been successfully applied to the study of lattice distortions ${ }^{5,6.7}$, diffuse defect scartering ${ }^{8.9}$, structural phase transitions $^{10-15}$ and charge density studies ${ }^{2}$. A special application is non-resonant magnetic scattering ${ }^{16-19}$. For high photon energies the magnetic cross section is only proportional to the squared Fourier transformation of the spin component perpendicular to the scattering plane ${ }^{16}$, which allows the study of the pure spin contribution to the magnetic moment ${ }^{14}$.

All existing high energy diffractometers scatter in the horizontal plane, because the polarization factor is negligible and heavy sample environments are easier to handle in the horizontal plane. This is an acceptable solution for undulator beam 
lines with a highly collimated beam and for wiggler beam lines with a short distance of the instrument to the source. In these cases the flux on the sample is high, even without beam focussing devices. Because the resolution of a diffractometer is also determined by the divergence of the incident beam ${ }^{20,21}$, focussing of the beam in the scattering plane should be avoided. At the Advanced Photon Source (APS) the distance from source to instrument is in general large. The elliptical multipole wiggler (EMW) produces photons with a high critical energy in a strongly divergent beam ${ }^{22}$. To collect the available intensity a focussing monochromator in the horizontal scattering plane is planned. In order to maintain high instrumental resolution the diffractometer will scatter in the vertical plane taking advantage of the small vertical divergence.

The outline of this contribution is as follows: First the general concept of a triple crystal diffractometer for high photon energies is introduced. After the introduction of the characteristics of the EMW insertion device, the choice of the first monochromator is discussed. First results in the search for a proper monochromator crystal are shown. At the end the principal design and properties of the planned diffractometer are presented.

\section{Diffractometer for high photon energies}

The basic concept of a triple crystal diffractometer is the elastic scattering with three crystals as shown in Fig. 1.

\section{monochromator}

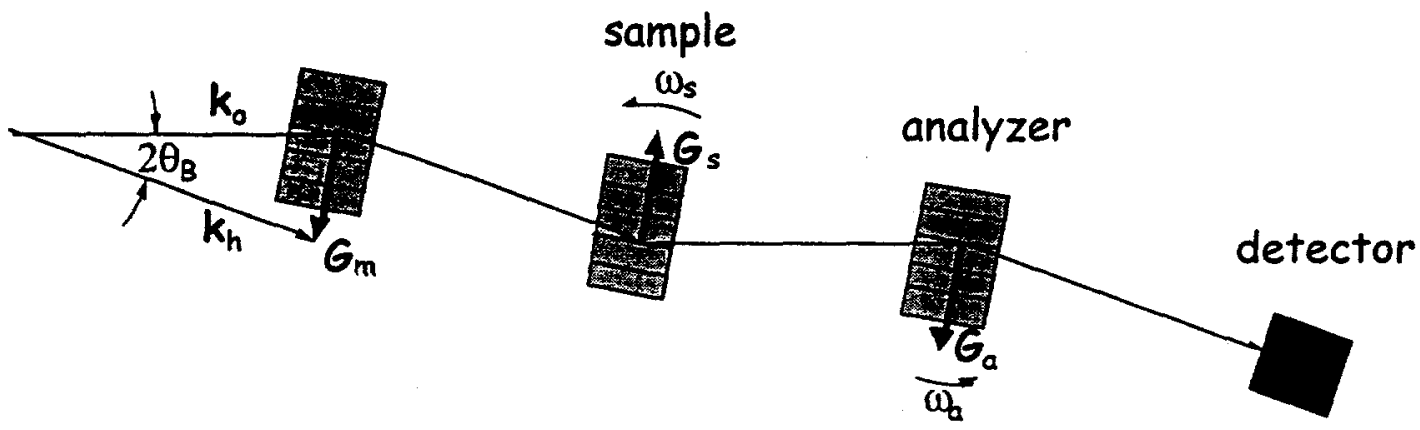

Fig. 1: Schematic drawing of a triple crystal diffractometer operated in Laue geometry. The monochromator determines the scattered wave vector $k_{0}\left(\left|k_{0}\right|=\left|k_{B}\right|\right)$ out of the white divergent beam. The Bragg angle $\theta_{B}$ for high photon energies is typically on the order of $1^{\circ}-3^{\circ}$ for fundamental reflections. The combination of different tilt angles of sample $\left(\omega_{5}\right)$ and analyzer $\left(\omega_{3}\right)$ crystals allows an investigation of the sample properties in the scattering plane in reciprocal space. In a non-dispersive setup, as shown here, the length of all three reciprocal scattering vectors $\mathbf{G}$ are the same.

The monochromator selects the scattered wave vectors out of a divergent white beam. The second crystal is the sample to be studied. The analyzer crystal operates like a slit and separates certain wave vectors $k$ scattered by the sample. The tilt angles $\omega_{3}$ and $\omega_{2}$ of sample and analyzer crystal are correlated to different positions in the reciprocal space around the reciprocal lattice point being investigated, see Fig. 2 . The transformation from angular space to the position in reciprocal space is given by $\Delta q_{x}=k \cos \theta_{B} \omega_{a}$ and $\Delta q_{y}=G_{s}\left(\omega_{k} / 2-\omega_{s}\right)$ parallel and perpendicular to the scattering vector $\mathbf{G}_{s}$, respectively ${ }^{20}$.

The instrumental resolution is defined for the case of three perfect crystals. The rocking curve of a perfect crystal decreases with increasing photon energy. For $100 \mathrm{keV}$ photons scattered by Si 220 the width of a perfect crystal rocking curve is $F W H M=0.4$ arcsec $(1.8 \mu \mathrm{rad})$. The instrumental resolution for a non-dispersive setup, where the momentum transfer of all three crystals is the same, for $100 \mathrm{keV}$ photons and Si 220 reflections is $2 \times 10^{-4} \AA^{-1}$ parallel and $10^{-5} \AA^{-1}$ perpendicular to the scattering vector ${ }^{20}$. Perpendicular to the scattering plane the resolution is determined by the acceptance angle of the detector. In the more general setup, where the momentum transfer of the sample is different from that of the monochromator and analyzer crystals, the energy resolution of the monochromator determines the instrumental resolution in reciprocal space. The energy resolution is mainly determined by the divergence of the incident beam on the monochromator crysta $^{21}$. An acceptable divergence of the beam is determined by the resolution needed in a dispersive setup. Since a good "real" crystal has a rocking curve with a FWHM $=1 \operatorname{arcsec}-20 \operatorname{arcsec}(5 \mu \mathrm{rad}-100 \mu \mathrm{rad})$, the divergence of the beam should be of the same order to optimize flux and resolution.

The requirements of a triple crystal diffractometer for high photon energies are mainly determined by the small rocking curves. High precision of the rotation tables and high stability are required. These conditions can only be achieved 
with a constant temperature for the surroundings and control by angular encoders 2.4 . Because of the easy use of sample surroundings, allowing in situ studies, the sample stage should be able to carry heavy equipment up to about $200 \mathrm{~kg}^{2}$.

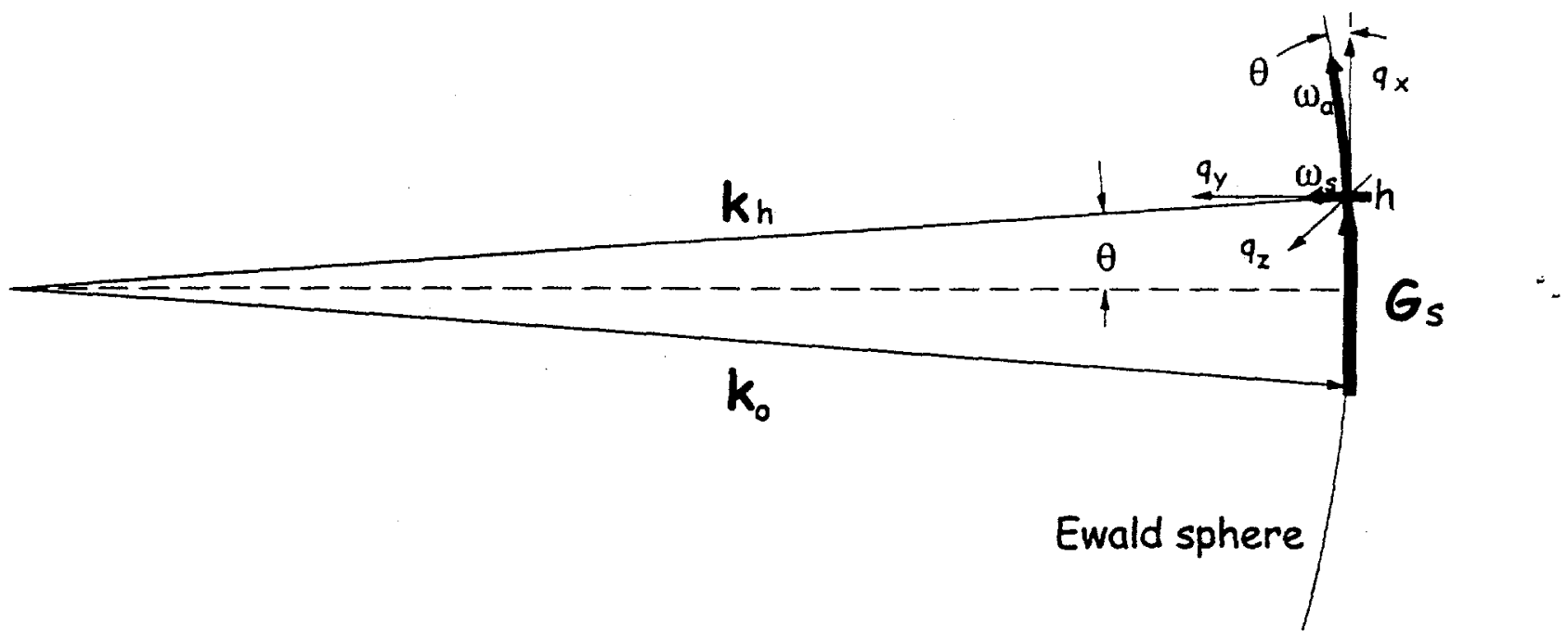

Fig. 2: Scanning geometry at the scattering vector of the sample for a triple crystal diffractometer. The origin of the coordinate system $q_{x}, q_{y}, q_{z}$ is defined at the reciprocal lattice point $h$. Tilting the sample around $\omega_{s}$ corresponds in reciprocal space to a tilt of the scattering vector $\mathbf{G}_{3}$. For small rotation angles a sample scan can be considered as a transversal scan along $\mathrm{q}_{\mathrm{y}}$. The rotation $\omega_{\mathbf{z}}$ of the analyzer crystal can be compared with a tilting of the scattered wave vector $\mathrm{k}_{\mathrm{b}}$, which leads to a movement along the Ewald sphere. Because of the small Bragg angle of about $2^{\circ}$ an analyzer scan almost corresponds to a longitudinal scan along $q_{\mathbf{x}}$.

\section{Insertion device}

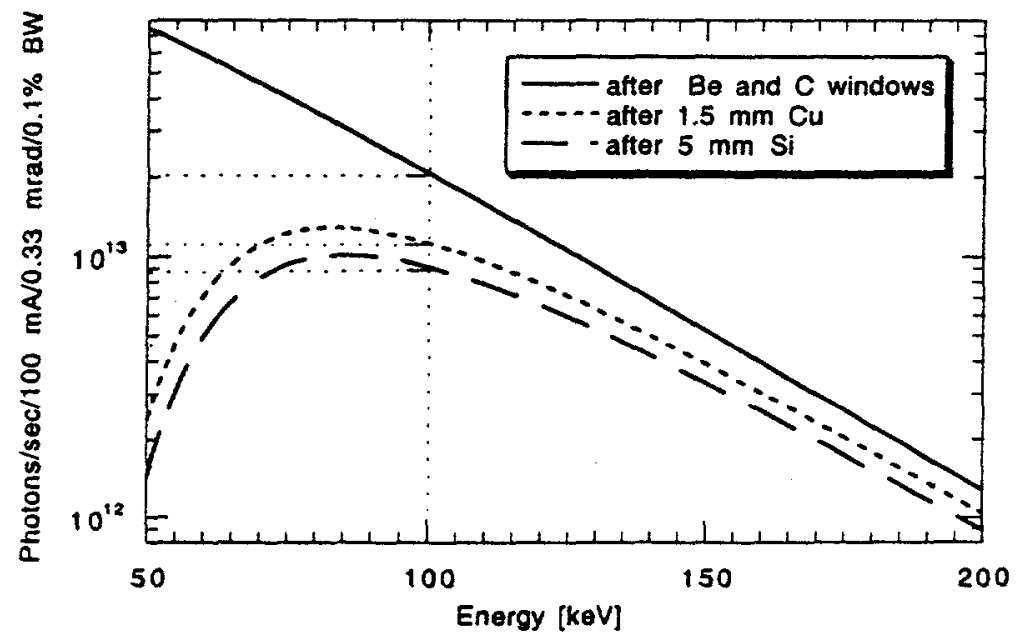

Fig. 3: Calculated flux from the EMW insertion device at the APS in $0.33 \mathrm{mrad}$ horizontal and full integration perpendicular to the storage ring plane. The vertical divergence of the beam is $1 / \gamma=73 \mu \mathrm{rad}$. The standard windows in the beamline are $1 \mathrm{~mm} \mathrm{Be}$ and $1 \mathrm{~mm} \mathrm{C}$. Because of the high penetration power of $100 \mathrm{keV} \mathrm{X}$-rays, a copper filter can be used in front of the monochromator to reduce the heat load on the crystal. The dashed curve shows the expected intensity after a $5 \mathrm{~mm}$ thick silicon crysta ${ }^{23}$.

The Basic Energy Sciences Synchrotron Radiation Center-Collaborative Access Team (BESSRC-CAT) has equipped sector 11 with an elliptical multipole wiggler as an insertion device ${ }^{22}$. This insertion device produces a photon spectrum with a critical energy of $32 \mathrm{keV}$. The radiated synchrotron beam has a divergence of $2 \mathrm{mrad}$ in the ring plane and $73 \mu \mathrm{rad}$ in the vertical plane. This device is able to produce circular polarized light on axis. In this case the high intense linearly polarized light will be above and below the ring plane. The beam will be used by three experimental stations at the same time. The high energy diffraction experiment uses the upper cone with mainly linearly polarized light. An energy spectrum for the expected 
intensities is shown in Fig. 3. The spectrum is calculated for the case, where only $16.5 \%$ of the horizontal beam size can be scallered by the munochromator, this is a realistic estimate for the initial operations of the beamline. In the final setup it is planned to use 1 mrad of the horizontal divergence.

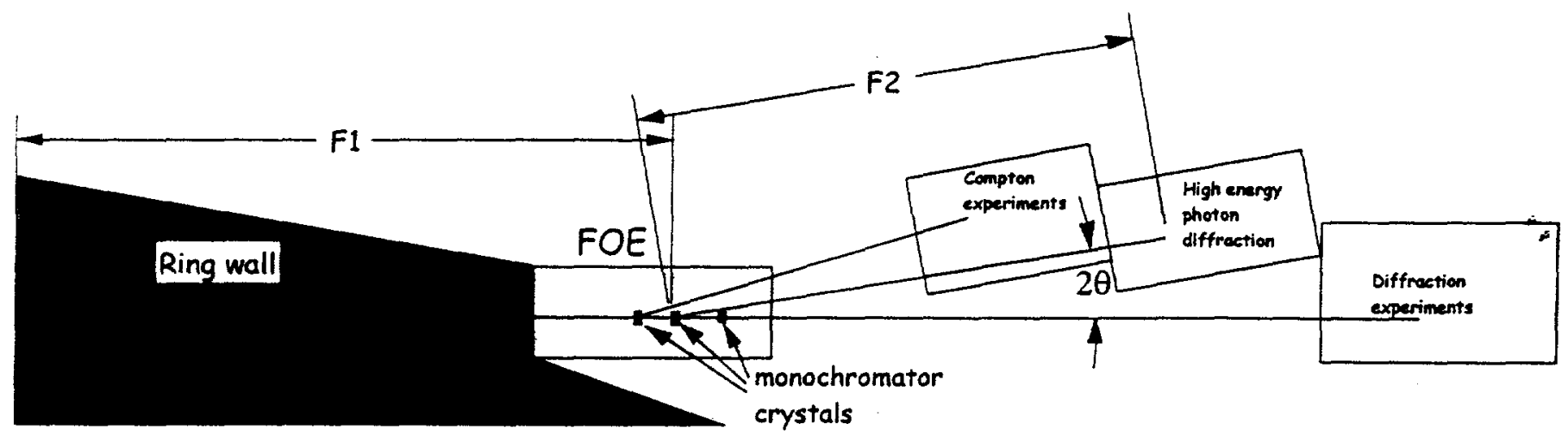

Fig. 4: Sketch of the floor plan of the EMW beamlines. The monochromators in the First Optical Enclosure (FOE) scatter the beam into the experimental stations. For the high energy photon diffraction station the angle $2 \theta$ is $3.8^{\circ}$. The distance $F 1$ from the source, the center of the EMW, to the monochromator is about $32 \mathrm{~m}$, the distance $F 2$ from monochromator to the experimental hutch about $23 \mathrm{~m}$.

A sketch of the floor plane is given in Fig. 4. The monochromators for all three stations are located in the first optical enclosure. The monochromator for the high energy X-ray diffraction beamline is $32 \mathrm{~m}$ downstream of the center of the insertion device. The monochromatic light is scattered at a Bragg angle of $2 \theta=3.8^{\circ}( \pm 1 \%)$ into the experimental hutch. The distance from the monochromator to the diffractometer is about $23 \mathrm{~m}$. Using silicon as material for the monochromator only certain photon energies in a range of $\pm 1 \%$ can be scattered into the experimental station. For the strongest reflections of Si the possible scattered photon energies are: $60 \mathrm{keV}$ (Sil11), $97 \mathrm{keV}$ (Si220), $114 \mathrm{keV}$ (Si311) and $138 \mathrm{keV}$ (Si400).

\section{Optic for high energies}

Up to now diffraction experiments with high energy synchrotron radiation have only been done using simple monochromator crystals. Mainly these crystals are scattering in Laue geometry. To increase the flux an imperfect crystal can provide more intensity on the sample. This intensity is paid for by a decrease in the instrumental resolution. The required instrumental resolution is determined by the quality of the sample. As imperfect monochromator crystals annealed silicon ${ }^{2,24,25}$ with FWHM $\leq 10 \operatorname{arcsec}(50 \mu \mathrm{rad}), \mathrm{Si}\left(\mathrm{TaSi}_{2}\right)^{26}$ with $\mathrm{FWHM} \leq 100 \operatorname{arcsec}(500 \mu \mathrm{rad}), \mathrm{SrTiO}_{3}{ }^{2}$ with a FWHM $\approx 40$ arcsec $(200$ $\mu \mathrm{rad})$ and $\mathrm{Si}_{1-x} \mathrm{Ge}_{x}{ }^{3}$ gradient crystals with $\mathrm{FWHM}=20 \mathrm{arcsec}(100 \mu \mathrm{rad})$ have been used. The last of these crystals is special, because in this crystal the lattice parameter is positional dependent. Annealed silicon crystals are interesting because of their relatively small and tunable mosaicity. The mosaicity matches the mosaicity of most good sample crystals and is therefore the best solution for many experiments.

As discussed in the previous section the triple crystal diffractometer will be about $55 \mathrm{~m}$ away from the source. The vertical divergence of the beam will give a beam height of about $4 \mathrm{~mm}$. Given the horizontal divergence of the beam ( $2 \mathrm{mrad})$ the width of the beam would be $110 \mathrm{~mm}$. Because a typical sample size is about $1-4 \mathrm{~mm}$, only $1-4 \%$ of the beam could be used. Focusing of high energy photons with a bent perfect silicon crystal scattering in Bragg geometry has been successfully used for spectroscopy experiments. The theory of bent monochromators and some applications are given in Ref. 27-29. A bent crystal focuses monochromatic photons onto the sample. The bending radius $R$ is given by the distance $F_{1}$ of source to monochromator, the distance $F_{2}$ from monochromator to sample, the Bragg angle $\theta$ and the asymmetric cut angle $\chi$ :

$$
R=2 F_{1} F_{2} /\left(F_{1} \cos (\chi-\theta)-F_{2} \cos (\chi+\theta)\right)
$$


In the symmetric Bragg case the angle $\chi$ is $90^{\circ}$, in the Laue case $0^{\circ}$. The energy resolution $\Delta \mathrm{E} / \mathrm{E}$ is for large bending radii and thin crystals determined by

$$
\Delta E / E=\cot (\theta) \Delta\left(1 /(R \cos (\chi+\theta))+1 / F_{1}\right)
$$

where $\Delta$ is the divergence of the incident beam. For a fixed Bragg angle and distances $F_{1}$ and $F_{2}$, the energy resolution is determined by $\chi$, see Fig. 5 . The bending radius $R$ follows from the optimization of the energy resolution.

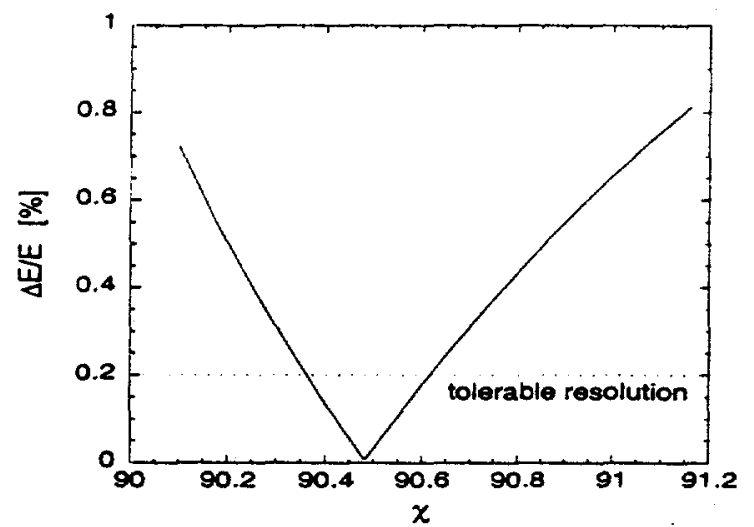

Fig. 5: Calculation of the energy resolution $\triangle E / E$ for $F_{1}=32 \mathrm{~m}, \mathrm{~F}_{2}=23 \mathrm{~m}$ and $\theta=1.9^{\circ}$ for different asymmetry angles $\chi$. The best energy resolution can be obtained at $\chi=90.48^{\circ}$. For a diffraction experiment a energy resolution of $\Delta \mathrm{E} / \mathrm{E}=0.2 \%$ is tolerable, so that the precision of the cut has to be only $\pm 0.1^{\circ}$

The reflectivity of a crystal can be increased by bending. Because of bending, the crystal can be described as a sum of independent perfect lamellas of small perfect crystals ${ }^{29}$. At a certain critical bending radius the size of the lamella is smaller than the extinction length. In this case the reflectivity of the whole crystal is not given by dynamical theory any longer. The critical bending radius $R_{\text {crit }}$ can be calculated by

$$
\mathrm{R}_{\mathrm{erit}}=\pi / 2 \cos (\theta) \mathrm{V}_{\mathrm{c}}^{2} \sin (2 \theta) / \lambda^{3} /\left(\mathrm{r}_{\mathrm{c}} C \mathrm{~F}_{\mathrm{b}}\right)^{2}
$$

where $V_{c}$ is the volume of the unit cell, $\lambda$ is the wavelength, $r_{e}=2.8510^{-15} \mathrm{~m}$ is the electron scattering length, $C$ is the polarization factor and $F_{h}$ is the structure factor ${ }^{27}$. For Si 220 at $100 \mathrm{keV}$ the critical radius is ca. $34 \mathrm{~m}$. The bending radius $R$ is $840 \mathrm{~m}$ for the EMW beamline calculated with the same numbers used for Fig. 5 and $\chi=90.5^{\circ}$. Therefore, at the EMW beamline bending will not provide an increase in the reflectivity.

The beam scattered by a bent monochromator is highly monochromatic, but strongly divergent. This is no problem for a spectroscopy experiment, but for a diffraction experiment the subsequent crystals can only accept the divergence, which is comparable to their rocking curves (5-100 $\mu \mathrm{rad}$ for good samples). In general a bent monochromator cannot be used in the scattering plane of the diffractometer. Therefore, the diffractometer at the EMW beamline has to scatter in the vertical scattering plane. In this case the beamline can fully utilize the flux collected by the bent monochromator and retain high instrumental resolution because of the small vertical divergence of $73 \mu \mathrm{rad}$.

Because the bending radius for our geometry is larger than the critical radius, an imperfect silicon crystal will be used to increase the reflectivity. An annealed Cz-grown silicon crystal is the only imperfect crystal, which is available in large enough lengths. The optimal mosaicity $\eta$ of the monochromator crystal is determined by the distance $F_{2}$ and the sample size, since a mosaic crystal will spread out the incident beam in $2 \times \eta^{30}$. For $F_{2}=23 \mathrm{~m}$ and $\eta=10 \operatorname{arcsec}(48 \mu \mathrm{rad})$ the beam width would be increased by $2.2 \mathrm{~mm}$. Considering a perfect crystal with a rocking curve width of $0.4 \operatorname{arcsec}(1.8 \mu \mathrm{rad})$, a gain of about a factor of 25 in the integrated intensity should be obtained by the use of a mosaic crystal with $\eta=10$ arcsec.

The mosaicity of an annealed crystal is determined by the quality of the $\mathrm{Cz}$-grown silicon and the annealing process. To find the right annealing process for a certain ingot an in situ study of different annealing processes using sample crystals from the ingot is necessary. The annealing process for an ingot was studied at HASYLAB at DESY using $100 \mathrm{keV}$ synchrotron radiation and a high temperature furnace ${ }^{31}$. This ingot can be annealed to a mosaicity of up to 11 arcsec $(53$ $\mu \mathrm{rad})$. The mosaicity of an annealed $\mathrm{Si}$ crystal is in principal a volume effect. A simple model is the Darwin mosaic model ${ }^{32}$. which considers a mosaic crystal to be built up out of small perfect crystallites, which are tilted with respect to each other, see Fig. 6. 


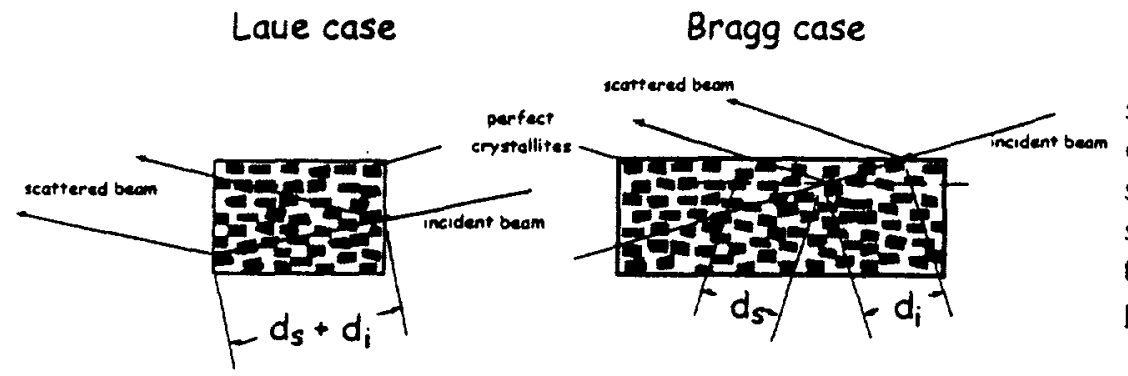

Fig.6 : Path $d_{i}$ and $d_{\text {, }}$ of the incident beam in symmetric Laue and Bragg cases. In the Laue case the beam penetrates the whole crystal. The sum of the path length of the incident and scattered beams is constant. In the Bragg case the path length in the crystal is dependent on the penetration depth of the incident beam.

To test if the annealing process is easy reproducible, a further sample $9 \times 11 \times 110 \mathrm{~mm}^{3}$ was annealed at Argonne National Laboratory. Moreover, it was important to prove that the mosaic crystal also works in the Bragg geometry for 100 $\mathrm{keV}$ photon energy. This sample was studied with a temporary setup at the EMW beamline using $100 \mathrm{keV}$ synchrotron radiation and perfect monochromators. The experimental setup is shown in Fig. 7.
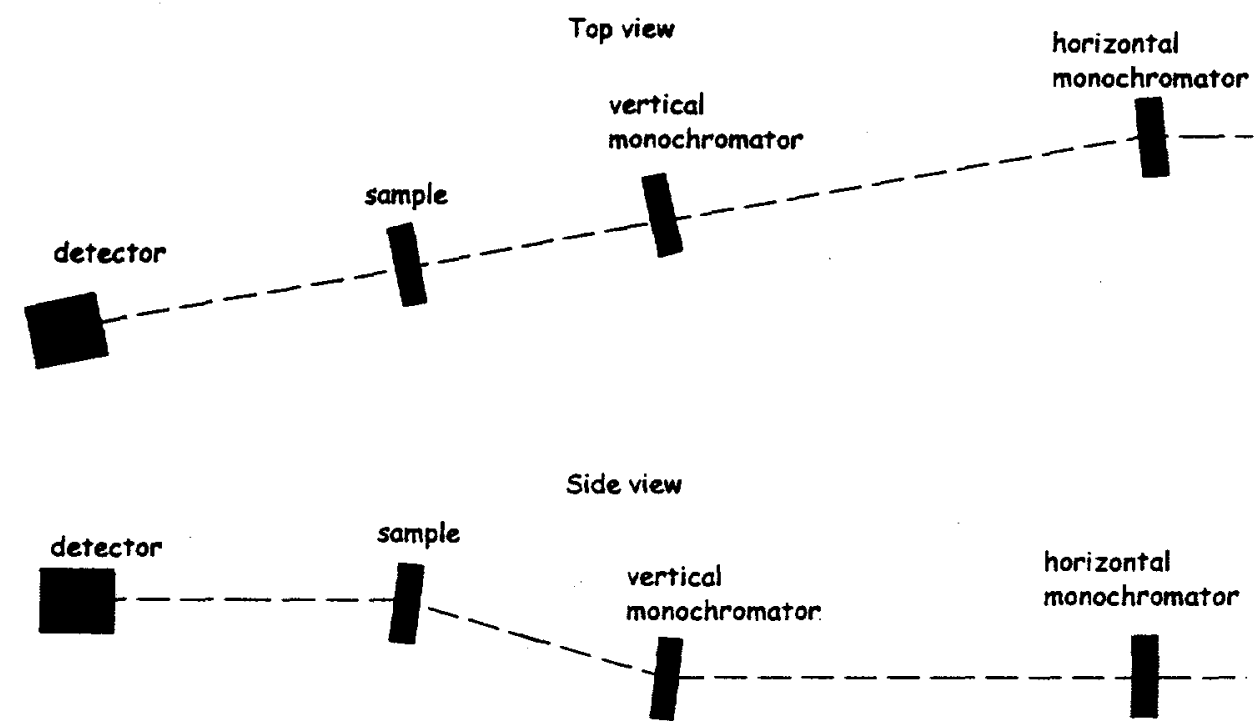

Fig. 7: Schematic drawing of the test setup. The first monochromator is a perfect silicon crystal scattering $100 \mathrm{keV}$ photons at the 440 reflection in the horizontal scattering plane. The second monochromator is $18 \mathrm{~m}$ downstream located in the experimental hutch. This crystal is also a perfect crystal scattering at the 220 reflection in the vertical plane. Both monochromators are used in Laue geometry. The sample is an annealed silicon crystal scattering at the 220 reflection in the vertical plane. If the sample crystal is oriented in the Laue geometry (as shown here), the beam has to penetrate ca. 9 mm of silicon. The crystal is also long enough $(110 \mathrm{~mm})$ to be used in Bragg geometry (compare Fig. 6).

The mosaicity, reflectivity, absorption length and the integrated intensity in Bragg and Laue geometries were studied. In both cases the photons were scattered at the 220 reflection. In Laue geometry 9 mm of silicon is penetrated. In the Bragg geometry the beam entered and exited on a $9 \mathrm{~mm} \times 110 \mathrm{~mm}$ long surface. The penetration of the crystal in this case is limited by the reflectivity and the absorption length, see Fig. 6 . To normalize the scattered intensity to the incident intensity on the sample the second monochromator was also scanned without the sample in the beam path immediately after the sample scans. Tilting the monochromator crystals corresponds to scanning of the beam divergence. The results are shown in Fig. 8.

The reflected peak intensity in Laue case is about $35 \%$ compared to the incident intensity on the sample. The mosaicity is given by the FWHM $=9.5 \operatorname{arcsec}(46 \mu \mathrm{rad})$ of the rocking curve. In the Bragg case the peak intensity is about 73 $\%$ and the mosaicity FWHM $=7$ arcsec (34 $\mathrm{rad}$ ). The most important number is the integrated intensity. A comparison of both cases is shown in Fig.9. The integrated intensity scattered in Bragg geometry is 1.7 times larger than that in the Laue case. To calculate the absorption of the sample in the Bragg case, the reflectivity of the sample was measured. For this 
purpose the detector is oriented to monitor the beam transmitted through the sample during a sample scan. In this geometry the intensity scattered by the sample cannot be detected, see Fig. 10.
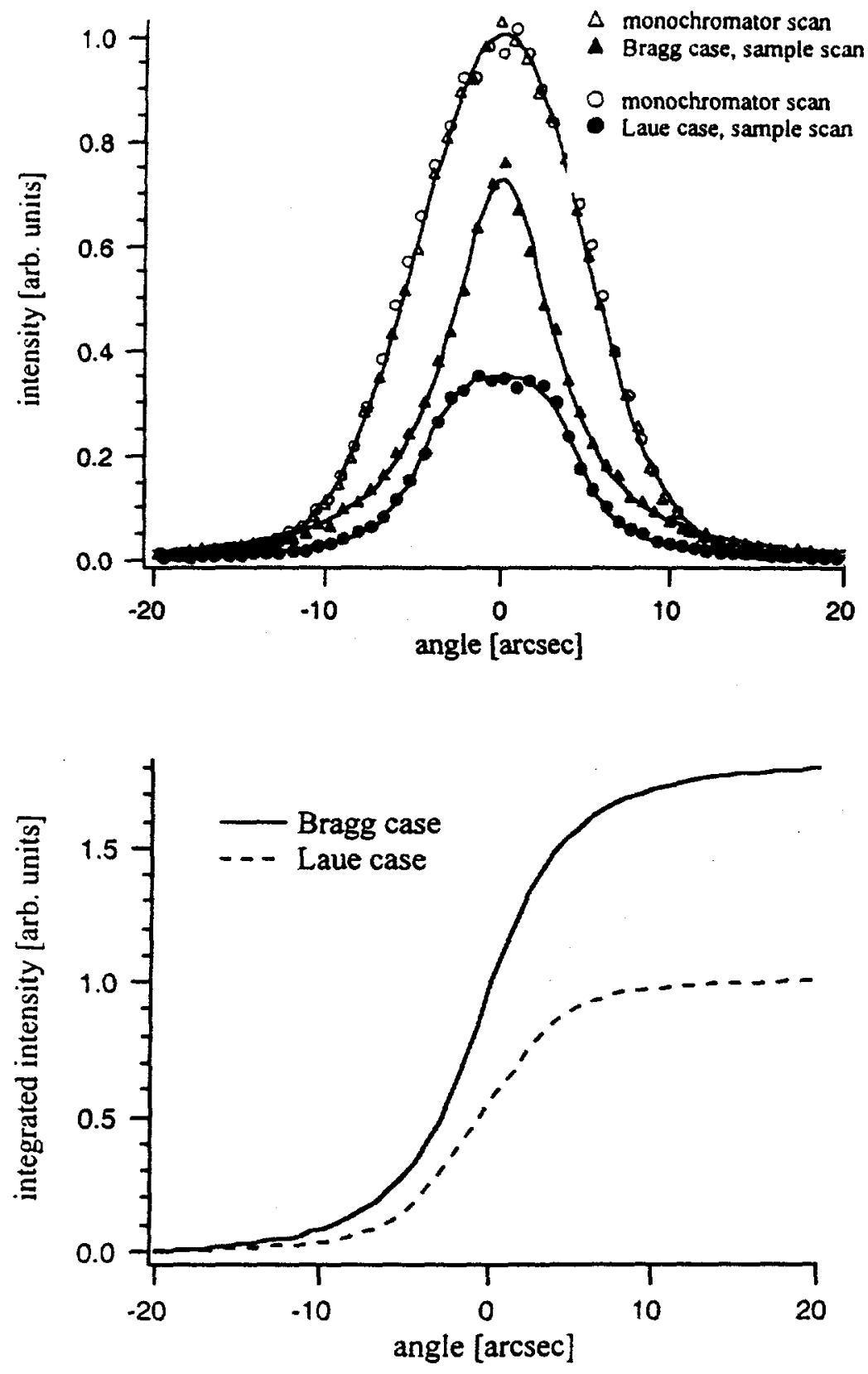

Fig.8: Comparison of a mosaic crystal in Bragg and Laue geometries. To normalize the scattered intensity in a sample scan, the incident intensity on the sample was checked by a monochromator scan. For this scan the sample was moved out of the beam path. The shape of monochromator scarn is determined by the divergence of the incident beam and can be fitted by a Gaussian with a FWHM of $60 \mu \mathrm{rad}$. The intensity of the sample scan is decreased by the reflectivity and the absorption of the sample. The profile of the scan is mainly determined by the mosaicity of the penetrated volume. In the Bragg case the peak intensity is $73 \%$ with a FWHM $=7$ arcsec. In the Laue case the peak intensity is $35 \%$, the FWHM $=9.5$ arcsec.
Fig. 9: Comparison of the integrated intensity in Bragg and Laue geometries. The integrated intensity in the Bragg case is about a factor of 1.75 times higher than in the Laue case. According to scattering theory there is a factor of 2 difference in intensity between the Bragg and Laue cases. The ratio here is smaller mainly because of the smaller mosaicity in the Bragg case.

In the Laue case the reflectivity is almost $50 \%$, which is the optimum according to theory ${ }^{32}$. In the Bragg case the reflectivity was determined to be $90 \%$. With the results shown in Fig. 8 the absorption of the crystal can be calculated. Considering a mean free beam path of $23 \mathrm{~mm}$ in silicon at $100 \mathrm{keV}$, the absorbed intensity in the Laue case corresponds to a thickness of $8.7 \mathrm{~mm}$ for the crystal. In the Bragg case the averaged beam path in the crystal is $5 \mathrm{~mm}$. As shown in Fig. 6 , only an averaged beam path of $2.5 \mathrm{~mm}$ contributes to the scattering intensity. This is different from the Laue case where the entire length contributes to the mosaicity.

Overall the scattering in Bragg geometry with the mosaic crystal was very successful. An annealed silicon crystal seems to be the proper solution to increase the integrated reflectivity. Compared to a perfect crystal an order of magnitude higher intensity can be expected. It is possible to cut a $270 \mathrm{~mm}$ long Si 220 and a $300 \mathrm{~mm}$ long Si 311 monochromator out of the tested ingot. These crystals will allow us to scatter and focus about $0.33 \mathrm{mrad}$ in the horizontal plane. 


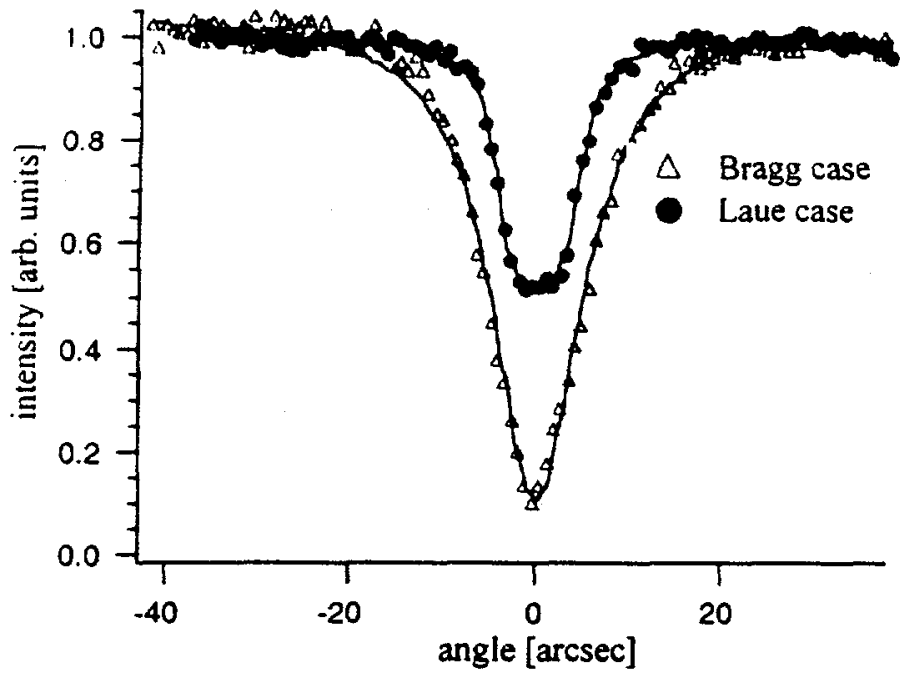

Fig. 10: Comparison of the reflectivity of the sample used in Bragg and in Laue geometries. The reflectivity is measured by the transmitted incident beam during a sample scan. The photons scattered by the sample cannot contribute to the detected intensity. In the Bragg case the reflectivity is $90 \%$, in the Laue case almost $50 \%$.

\section{The diffractometer at the APS}

The requirements of a triple crystal diffractometer for high energy X-rays diffraction at the APS are: high angular precision and stability, a vertical scattering plane to allow a focusing first monochromator, the ability to handle heavy sample environments on the sample stage and the flexibility allowing a study of high momentum transfers. The principle ideas in the design of a triple crystal diffractometer for high energy synchrotron radiation are given in the references 1 and 2 . The basic concept for this diffractometer shown in Figures 11 and 12 is as follows. The three crystals of the diffractometer have to be on a common optical table to guarantee the angular stability. The optical table will be supported on 4 feet with translations to align the diffractometer to the beam height. The whole table can be moved perpendicular to the beam to take advantage of the possibility of varying the photon energy around $\pm 1 \%$.

To allow higher flexibility the detector will be placed on an extra slide independent from the optical table. In this case it can be moved up to $3.5 \mathrm{~m}$ away from the analyzer to increase the resolution perpendicular to the scattering plane. The detector also can be moved close to the sample (about $1 \mathrm{~m}$ ) enabling the study of high momentum transfers. The detector also has a translation perpendicular to the beam. A long z-translation maintains the detector at the height of the beam, a rotation table keeps it in the right direction.

The three towers on the optical table can be moved on a common translation in direction of the beam (y). The goniometers for the monochromator and analyzer crystals will be identical. They will have three rotations to orient the crystal. The monochromator goniometer will be mount on a simple tower with a translation perpendicular to the scattering plane. The analyzer tower in addition has a long z-translation. The $\omega$-rotation of the analyzer will be controlled by an encoder to guarantee the position in the reciprocal space.

The sample tower must to be able to carry heavy sample environments up to $200 \mathrm{~kg}$. A large stage permits the ztranslation of the sample tower. The $\omega$-rotation table has to support two further rotation tables to orient the sample and two translations. The translations allow position dependent measurements in large sample crystals. The sample $\omega$-rotation will also be equipped with an encoder. To allow a higher degree of freedom for the sample orientation, on additional arm with a rotation table will be provided. This extra arm will be mounted on the top of the sample stage with an $\chi$ angle of $60^{\circ}$ to the surface. It can be regarded as a kind of $K$-goniometer.

The background in the beam path between the crystals will be suppressed by iron collimators and slit systems. This collimating system will by mount on telescope slits, which are automatically guided in the beam direction by a rotation connected with the z-translation of the towers.

The diffractometer will be flexible enough to enable the instrument to study momentum transfers up to $35 \AA^{-1}$. For samples with low crystal quality the monochromator tower for the second monochromator can be removed to gain more flux on the sample. For experiments without an analyzer crystal the analyzer stage can also be removed to allow a shorter distance between detector and sample tower. Because of the encoder, a controlled instrumental resolution of $10^{-4} A^{-1}$ in the scattering plane can be achieved. 


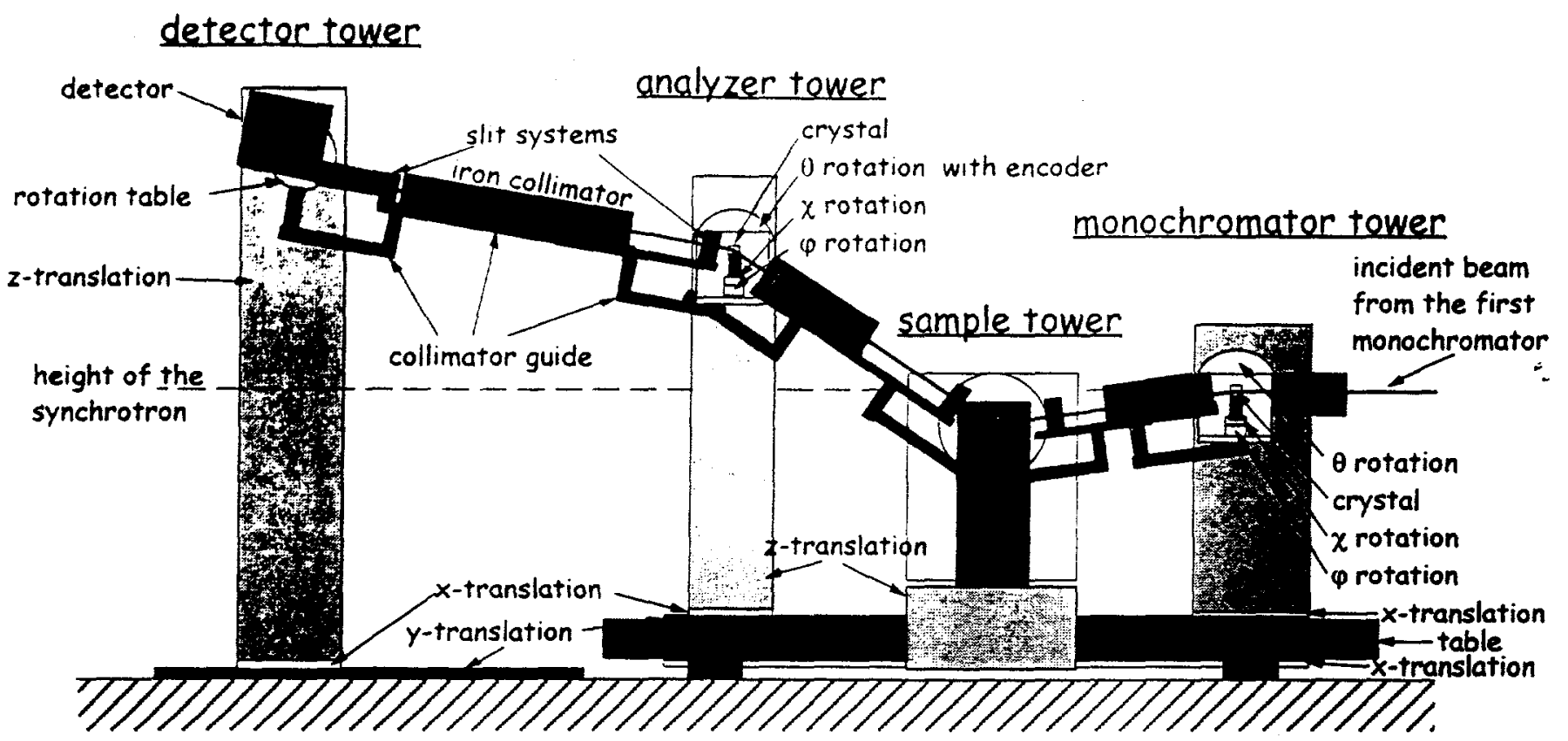

Fig. 11: Schematic drawing of the front view of the diffractometer planned for the APS. A further description of the diffractometer is in the text.

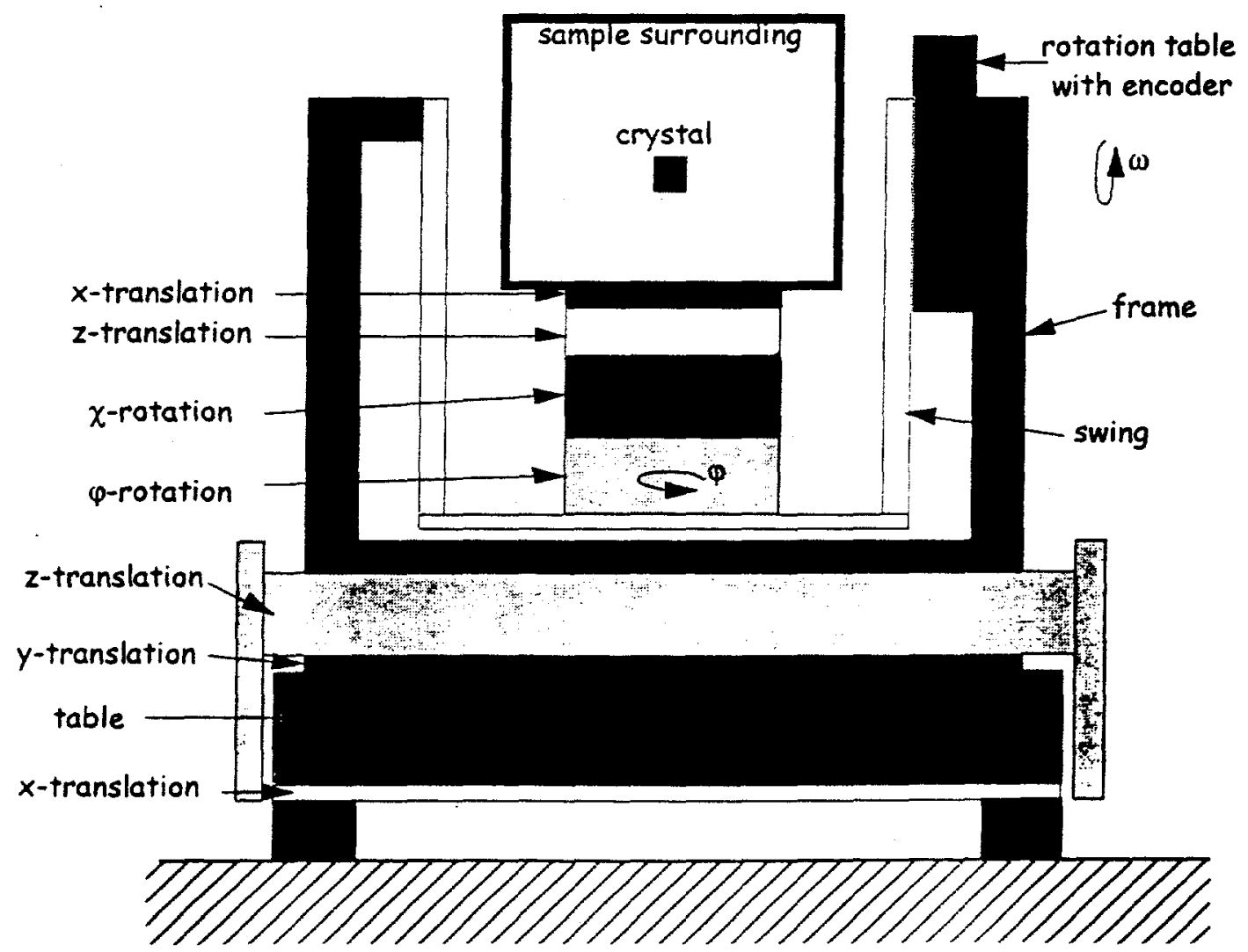

Fig. 12: Schematic drawing of the side view of the sample tower. A description can be found in the text. To be able to carry a heavy weight, the $\omega$-table has a counterpoint. The further rotation and translations of the sample are mount on this swing assemble. 


\section{Acknowledgment}

Work at Argonne National laboratory is sponsored by the US Department of Energy, Office of Basic Energy Sciences, Division of Materials Sciences, under contract W-31-109-ENG-38 and the State of Illinois under HECA.

\section{References}

1. J.B. Hastings, D.P. Siddons, J.R. Schneider and L.E. Berman, "Three-crystal Spectrometer for $150 \mathrm{keV}$ Synchrotron Radiation", Rev. Sci. Instrum. 60, 2398-2401, 1989.

2. R. Bouchard, D. Hupfeld, T. Lippmann, J. Neuefeind, H.-B. Neumann, H.F. Poulsen, U. Rütt, T. Schmidt, J.R. Schneider, J. SüBenbach and M. von Zimmermann, "A Triple-Crystal Diffractometer for High-Energy Synchrotron Radiation at the HASYLAB High-Field Wiggler Beamline BW5", J. Synchrotron Rad. 5, 90-101, 1998.

3. T. Kracht, J.R. Schneider, H. Schulte-Schrepping and K. Jønson, "PETRA Undulator beamline: Experimental Stations", HASYLAB Annual Report 1995, 151-159.

4. K.-D. Liss, A. Royer, T. Tschentscher, P. Suortti and A.P. Williams, "On High-Resolution Reciprocal-Space Mapping with a Triple-Crystal Diffractometer for High-Energy X-rays", J. Synchrotron Rad. 5, 82-89, 1998

5. S. Keitel, C.C. Retsch, T. Niemöller and J.R. Schneider, "Si ${ }_{1-x} G_{e}$ Gradient Crystals: A new Monochromator Material for Hard X-rays", submitted to Nucl. Instr. and Meth. A

6. A. Magerl, K.-D. Liss, J.B. Hastings, D.P. Siddons, H.-B. Neumann, H.F. Poulsen, U. Rütt, J.R. Schneider and R. Madar, "The Local Perfection of Massive Gradient Crystals Studied by High-energy X-ray Diffraction", Europhys. Lett. $31,5,329-334,1995$.

7. K.-D. Liss, A. Magerl, A. Remhof and R. Hock, "Ultrasound-induced Gradient Crystals Observed by High-energy Xrays", Acta Cryst. A53, 181-186, 1997.

8. R. Bouchard, S. Kouptsidis, H.-B. Neumann, T. Schmidt, J.R. Schneider, "Non-imaging Characterization of Imperfect Crystals by Means of a Three-crystal Diffractometer for High Energy Synchrotron Radiation", J. Appl. Phys. 73, 36803684, 1993.

9. T. Schmidt, D. Woo, S. Keitel, J.R. Schneider, U. Lambert and W. Zulehner, "Effect of Thermal Diffuse Scattering in Triple-Crystal Diffractometry with High-Energy Synchrotron Radiation", accepted by J. Appl. Cryst.

10. H.-B. Neumann, H.F. Poulsen, U. Rütt, J.R. Schneider and M. von Zimmermann, "Investigation of Structural Phase Transitions in Perovskites Using High Energy Synchrotron Radiation", Phase Trans. 55, 17-35, 1995.

11. H.-B. Neumann, U. Rütt, J.R. Schneider and G. Shirane, "Origin of the Critical Scattering on Two Length Scales in $\mathrm{SrTiO}_{3}$ : A High-Energy Synchrotron-Radiation diffraction study", Phys. Rev. B 52, 3981-3984, 1995.

12. U. Rütt, A. Diederichs, J.R. Schneider and G. Shirane, "Depth Dependence of Strain, Mosaicity and Sharp Component in the Critical Scattering of SrTiO3.", Europhys. Lett. 39, 395-400, 1997.

13. H.F. Poulsen, M. von Zimmermann, J.R. Schneider, N.H. Andersen, P. Schleger, J. Madsen, R. Hadfield, H. Casalta, R. Liang, P.Dosanjh, W. Hardy, "Structural Phase Transition in Bulk $\mathrm{YBa}_{2} \mathrm{Cu}_{3} \mathrm{O}_{6+\mathrm{x}}$ with $\mathrm{x}=0.35$ and $\mathrm{x}=0.36$ ", Phys. Rev. B 53, $15335-15344,1996$.

14. A. Vigliante, M. von Zimmermann, J.R. Schneider, T. Frello, N.H. Andersen, J. Madsen, D.J. Buttrey, D. Gibbs and J.M. Tranquada, "Detection of Charge Scattering Associated with Stripe Order in $\mathrm{La}_{1.775} \mathrm{Sr}_{0.225} \mathrm{NiO}_{4}$ by Hard X-ray Diffraction", Phys. Rev. B 56, 8248-8251, 1997.

15. T. Niemöller, M. von Zimmermann, J.R. Schneider, S. Uhlenbruck, O. Friedt, B. Büchner, T. Frello and N.H. Andersen, "The Charge Ordered Phase in $\mathrm{La}_{1-x} \mathrm{Sr}_{\mathbf{x}} \mathrm{MnO}_{3}$ Studied by Means of Hard X-ray Diffraction", submitted to Eur. J. Phys.

16. M. Lippert, T. Brückel, T. Köhler and J.R. Schneider, "High Resolution Bulk Magnetic Scattering of High Energy Synchrotron Radiation", Europhys. Lett. 27, 537-541, 1994.

17. J. Strempfer, T. Brückel, U. Rütt, J.R. Schneider, K.-D. Liss and T. Tschentscher, "The Non-Resonant Magnetic X-ray Scattering Cross Section of $\mathrm{MnF}_{2}, 2$. High-Energy X-ray Diffraction at $80 \mathrm{keV",} \mathrm{Acta} \mathrm{Cryst.} \mathrm{A52,} \mathrm{438-449,} 1996$.

18. J. Strempfer, Th. Brückel, D. Hupfeld, J.R. Schneider, K.-D. Liss and Th. Tschentscher,"The Non-resonant Magnetic Xray Scattering Cross-section for Photon Energies up to $500 \mathrm{keV}$ ", Europhys. Lett. 40, 569-574, 1997

19. J. Strempfer Th. Brueckel, W.Caliebe, G.J. Mclntyre, F. Tasset, Th. Zeiske, K. Burger, W. Prandl and J.R. Schneider, "Form-Factor Measurements on Chromium with High-Energy Synchrotron Radiation and Neutrons", to be published

20. H.-B. Neumann. U. Rütt, R. Bouchard and J.R. Schneider. "The Resolution Function of a Triple-Crystal Diffractometer for High-Energy Synchrotron Radiation in Non-dispersive Laue Geometry", J. Appl. Cryst. 27, 1030-1038, 1994. 
21. U. Rütt, H.-B. Neumann, H.F. Poulsen and J.R. Schneider, "The Resolution Function of a Triple-Crystal Diffractometer for High-Energy Synchrotron Radiation. II. Dispersive Laue Geometry", J. Appl. Cryst. 28, 729-737, 1995.

22. P.A. Montano, G.S. Knapp. G. Jennings, E. Gluskin, E. Trakhtenberg, I.B. Vasserman, P.M. Ivanov, D. Frachon, E.R. Moog, L.R. Turner, G.K. Shenoy, M.J. Bedzyk, M. Ramanathan, M.A. Beno and P.L. Cowan, "Elliptical Multipole Wiggler Facility at the Advanced Photon Source", Rev. Sci. Instr., 66, 1839-1841, 1995.

23. D. Chapman, W. Thomlinson, N. Gmur and N. Lazarz, "Photon-A Program for Synchrotron Radiation-Dose Calculations", Nucl. Instr. and Meth. A266, 191, 1988.

24. J.R. Schneider, O.D. Gonçalves, A.J. Rollason, U. Bonse, J. Lauer and W. Zulehner, "Annealed Czochralski Grown Silicon Crystals: A New Material for the Monochromatisation of Synchrotron Radiation and X-rays above $60 \mathrm{keV",}$ Nucl. Instr. and Meth., B29, 661-674, 1988.

25. J.R. Schneider, H. Nagasawa, L.E. Berman, J.B. Hastings, D.P. Siddons and W. Zulehner, "Test of Annedled Czochralski Grown Silicon Crystals as X-ray Diffraction Elements with $145 \mathrm{keV}$ Synchrotron Radiation", Nucl. Instr. and Meth. A276, 636-642, 1989.

26. H.-B. Neumann, J.R. Schneider, J. Süßenbach, S.R. Stock and Z.U. Rek," Si-TaSi2 In Situ Composites: a New Monochromator Material for Hard X-rays", Nucl. Instr. and Meth. A372, 551-555, 1996.

27. P. Suortti, U. Lienert and C. Schulze, "Focusing monochromators for high energy synchrotron radiation", Nucl. Instr. And Meth. A338, 27-32, 1994.

28. P. Suortti, D. Chapman, J.R. Schneider and T. Tschentscher, "Bent-Crystal Monochromator for 150 keV Synchrotron Radiation", J. Appl. Cryst. 25, 432-438, 1992.

29. E. Erola, V. Eteläniemi, P. Suortti, P. Pattison, W. Thomilson, "X-ray Reflectivity of Bent Perfect Crystals in Bragg and Laue Geometry", J. Appl. Cryst. 23, 35-42, 1990.

30. A. Freund, "Application of Mosaic Crystals of High Brilliance X-ray Optics", SPIE proceedings,1740, 58-69,1992

31. S. Keitel, U. Rütt, M.A. Beno and J.R. Schneider, to be published in HASYLAB Annual Reports, 1998

32. W.H. Zachariasen, Theory of $X$-ray Diffraction in Crystals, New York, Wiley, 1945. 